\title{
Dynamics of a Carriage System of Comparator for Calibrating the Line Standards of Length
}

\author{
A. Kilikevičius, V. Vekteris and V. Mokšin* \\ Department of Mechanical Engineering, Vilnius Gediminas Technical University, J. Basanavičiaus Str. 28, 03224 Vilnius, \\ Lithuania
}

Received 14 April 2014; Accepted 10 March 2015

\begin{abstract}
This paper presents research dynamic properties of a calibration comparator which is used to calibrate high precision line standards of length. For this purpose, multi-body dynamic and mathematical models of a carriage system of the comparator were presented. Calculated amplitude-frequency responses and modes of oscillations allowed a determination of resonant frequencies of the system.
\end{abstract}

Keywords: Vibration amplitude, Resonant frequency, Carriage, Line standard of length, Calibration comparator.

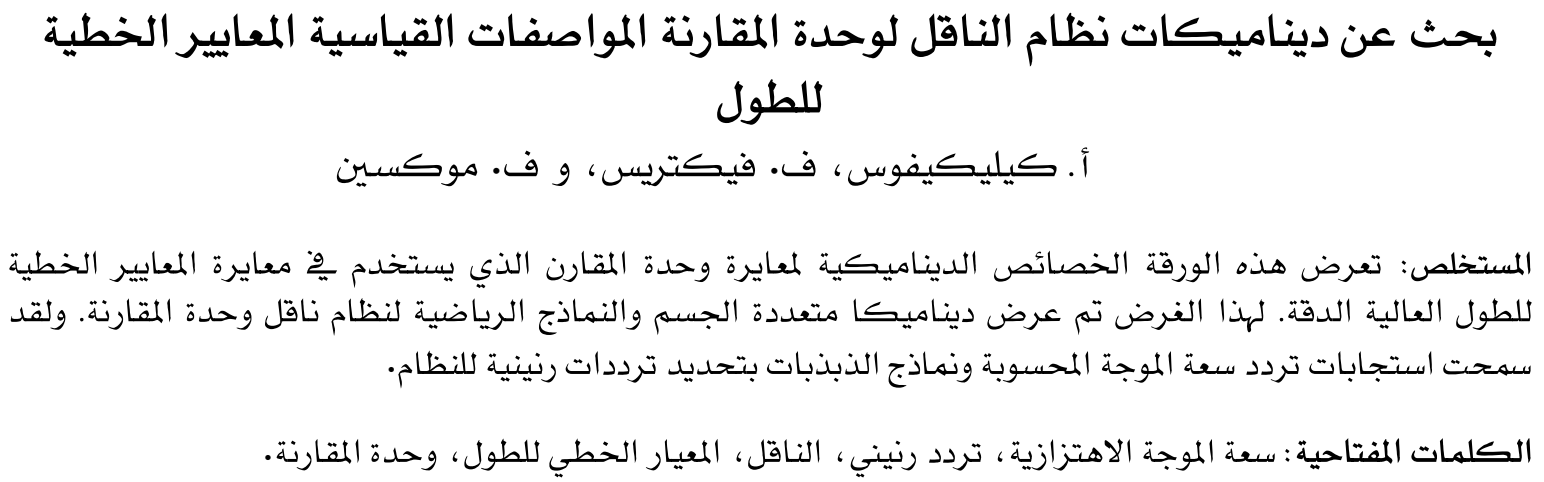

\section{Introduction}

Length calibration is one of the most complex, important, and fundamental issues facing science and high-tech industries. The meter is the most commonly used length measurement unit and the need for reliable, precise measurements to ensure unity of measurements worldwide constantly grows. Recently, linear displacement transducers increasingly have been used to precisely measure displacements, particularly in automated industrial equipment (Sawabe et al. 2004; Thalmann 1997). At present, line standards technology is well developed, and precise scales and gratings have replaced interferometers in displacement measuring systems (Demarest 1998; Jakstas et al. 2014; Kasparaitis et al. 2012; Kasparaitis et al. 2015).

*Corresponding author's email: Vadim.moksin@vgtu.it
The relevance of the accurate and fast detection of divisions of line standards of length is primarily determined by the constantly increasing requirements of precision scale calibration productivity (BIPM 2003; RAENG, 2004; Swyt 2001). The new generation of precision scale production and calibration systems must meet significantly higher accuracy and efficiency requirements (Swyt 2001; Webb and Jones 2004; Bosse and Flugge 2002; Kasparaitis et al. 2012). To fulfill these requirements, accuracy parameters of precision scales should be metrologically linked to those of higher etalons already in place during the manufacturing process. These needs can be satisfied by creating qualitatively new systems which reflect modern scientific and technological achievements and optimally meet the specific calibration requirements, as well as by improving existing calibration systems in accordance with the fundamental principles of precision engineering (Nakazava 1994). 
In designing precision length calibration systems which fulfill specific requirements, it is necessary to solve a number of new scientific and technical problems, in particular with regard to the calibration uncertainty. Modern scientific and technical achievements allow length measurements at a nano-level; therefore, a qualitative and quantitative description of the determinants of the precision calibration process requires complex mathematical models and the use of numerical methods. This is especially true for an evaluation of the influence of thermal and contact deformations on calibration accuracy, the compensation of geometrical and optical dynamic errors, the measurement signal processing, and so on.

It is known that two scale calibration techniques can be used in calibration comparators: static and dynamic. If the former method is used, the calibrating element usually is scanned many times; therefore, it is easy to accumulate measurement data and reduce the influence of random noise on calibration uncertainty. However, during the line scanning process, the scale position in respect to the microscope must be fixed. Therefore, the static method is potentially more accurate, but slower than the dynamic method. It is used in high precision calibration systems such as the NIST (USA), METAS (Switzerland), PTB (Germany) comparators, as well as in precise chip patterns comparators (Beers and Penzes 1999; METAS 2000). This method provides an edge detection accuracy of less than $1 \mathrm{~nm}$.

Recent progress in measurement technology allows precise calibration under dynamic conditions. In such a case, calibration is performed during the motion of a microscope or scale. The dynamic calibration method benefits are especially noticeable if long standards are calibrated to greater than 1 meter. The advantages of the latter method can be presented as follows:

- the calibration process is much faster (productivity increases);

- the process lends both a simple comparator's design and control, because it is not necessary to accurately capture the position of scale with respect to the microscope;

- the calibration accuracy is less influenced by a change of environmental conditions due to the reduced duration of the calibration procedure.

Despite the mentioned advantages, the dynamic regime of calibration can lead to dynamic calibration errors due to vibrations originating in the calibration system. Dynamic effects become extremely important because vibrations directly influence the reading accuracy. New demanding and contradictory requirements of high-speed and accurate precision line scale calibrations calls for an in-depth analysis of the noise and vibrations associated with drivers, dampers, and the moving parts of the calibration system (Jakstas et al. 2011).

This paper analyzes the dynamics of line standards in a length calibration comparator's carriage system in which a line detection system and interferometer mirrors were installed. Carriage vibration analysis allowed the establishment of harmful resonant frequency levels and an evaluation of the system's response to external forces.

\section{Research Objectives}

The length calibration comparator (Kasparaitis et al. 2008; Kaušinis et al. 2009; Kilikevičius et al. 2009; Kilikevičius and Sabaitis 2008) consists of nine main parts: a laser interferometer, gauges for measuring environmental parameters, a microscope with a CCD camera, a driver system, a controller, a data accumulating and processing computer, a compensation system, granite guides, and a carriage system consisting of the force and precision carriages [Fig. 2].

The base part of the comparator [Fig. 1] is massive-four meters in length with a granite frame with guides. The frame sits on four pneumatic supports, which dampen high frequency vibrations. Carriages are placed on rigidly mounted aerostatic supports and preloaded by means of springy supports mounted from the opposite sides. It enables an adjustment of required clearances in aerostatic supports and an increase in the rigidity of the system. The carriage system moves along the frame guides.

The carriage system is pulled along the guides by means of the program-controlled friction driver [Fig. 1]. The construction of the carriage and the driver system enables the elimination of the influence of driver errors on the accuracy of the longitudinal motion. To this aim, the carriage system is composed of the force and precision carriages [Fig. 2]. 


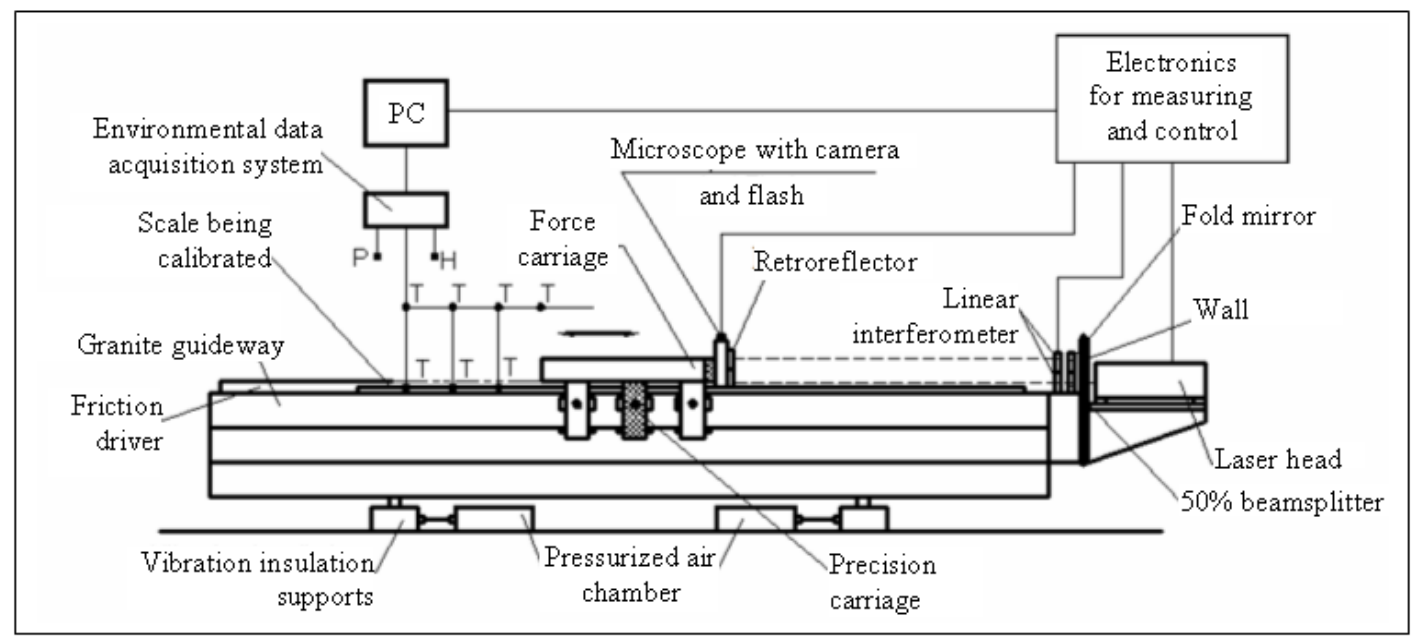

Figure 1. Scheme of the comparator.

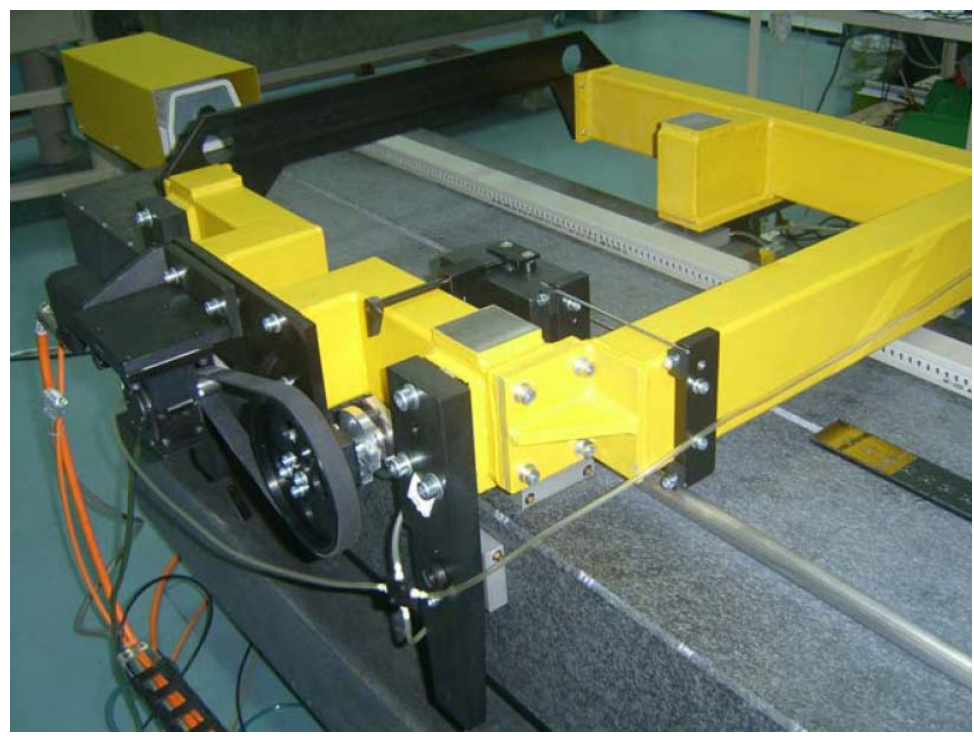

Figure 2. The force carriage of the comparator.

The driver is connected to the force carriage; however, all measuring systems used to measure carriage motion and detect the lines on the scale are connected to the precision carriage. The force and precision carriages are connected by a special force transmitting element. This element is designed in such a way that the direction of the force transmitted by it coincides with the direction of the motion of the carriages along guides, where forces in the transverse direction are not applied.

\section{Dynamic Modeling of the Carriage System}

Equations of motion can be derived by dividing the comparator model into two subsystems: the force carriage subsystem with links to the granite frame $\left(O_{1}\right)$ and the precision carriage subsystem with links to the granite frame and the force carriage $\left(\mathrm{O}_{2}\right)$ [Fig. 3]. The integration of subsystems into the whole carriage system is done by means of auxiliary links expressed using the auxiliary (surplus) coordinates. 


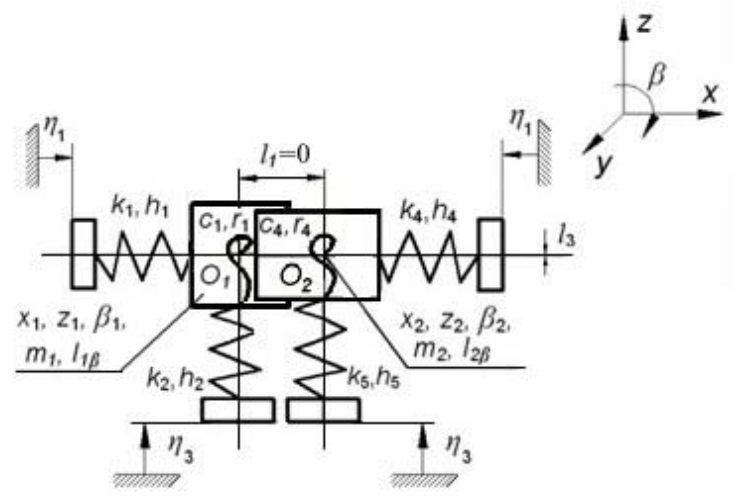

a)
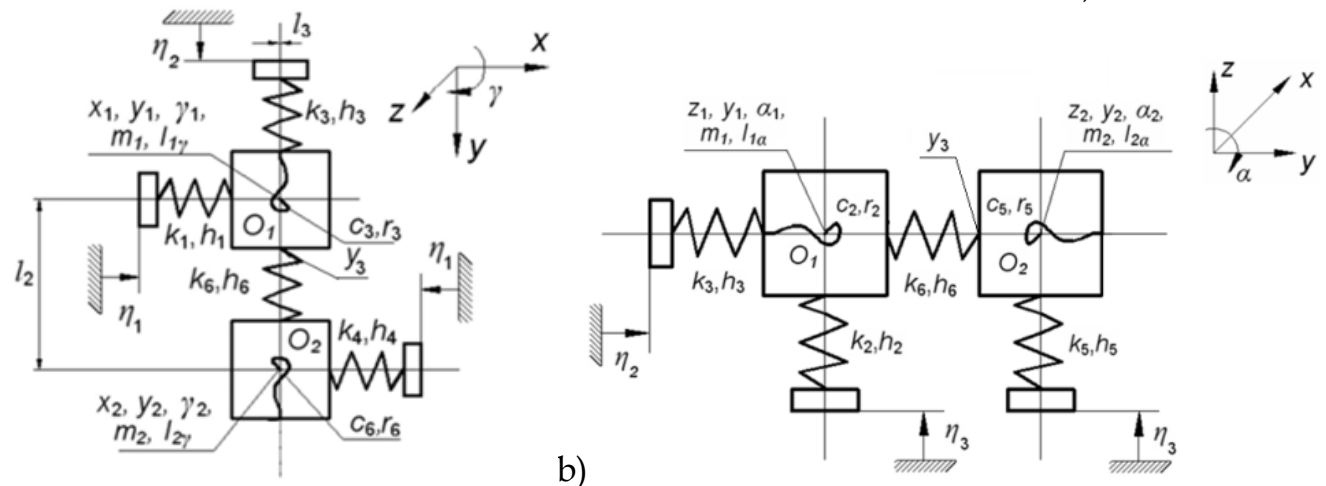

c)

Figure 3. A dynamic model of the carriage system of the comparator.

The views in Fig. 3 are: a) $x z$ plane, b) $y x$ plane, and c) $y z$ plane. $O_{1}$ and $O_{2}$ are the origins of the coordinate systems of separate subsystems of the model which coincide with mass centers of corresponding parts $m_{1}$ and $m_{2} . I_{1 j}, I_{2 j}$, are moments of inertia, where $j$ corresponds to angles $\alpha, \beta$, and $\gamma$. $k_{\mathrm{n}}$ are coefficients of rigidity in the direction of linear axes $(n=1,2, \ldots, 6)$, while $c_{n}$ are coefficients of rigidity in the direction of rotational axes $(n=1,2, \ldots$, $6), h_{\mathrm{n}}$ are damping coefficients in the direction of linear axes $(n=1,2, \ldots, 6)$, and $r_{n}$ are damping coefficients in the direction of rotational axes $(n=1$, $2, \ldots, 6) . \quad \eta$ expresses the kinematical excitation function, or oscillations of the foundation.

The comparator is complex dynamic system with many degrees of freedom, is elastic and dissipative [Fig. 3]. In order to derive differential equations describing the behavior of such objects, it is expedient to use the second order Lagrange equation (Augustaitis 2000) as appears in Eqn. 1, $\frac{d}{d t}\left(\frac{d T}{d\{\dot{q}\}}\right)-\frac{d T}{d\{q\}}+\frac{d \Phi}{d\{\dot{q}\}}+\frac{d \Pi}{d\{q\}}=\{Q(t)\}$

where $T, \Pi$ and $\Phi$ are the kinetic and potential energies; the dissipative function of the system, $\{q\},\{\dot{q}\}$, are the vectors of displacements and velocities, and $\{Q(t)\}$ is the vector of external excitation forces.

The kinetic and potential energy of individual subsystems-force carriage $O_{1}$ and precision carriage $\mathrm{O}_{2}-$ can be expressed as follows:

$2 T_{i}=m_{i}\left(\dot{x}_{i}^{2}+\dot{y}_{i}^{2}+\dot{z}_{i}^{2}\right)+I_{i \alpha} \dot{\alpha}_{i}^{2}+I_{i \beta} \dot{\beta}_{i}^{2}+I_{i \gamma} \dot{\gamma}_{i}^{2}, i=1,2$

$2 \Pi_{1}=k_{1}\left(x_{1}-x_{0}\right)^{2}+k_{2}\left(z_{1}-z_{0}\right)^{2}+$

$k_{3}\left(y_{1}-y_{0}\right)^{2}+c_{2}\left(\alpha_{1}-\alpha_{0}\right)^{2}+c_{1}\left(\beta_{1}-\beta_{0}\right)^{2}+c_{3}\left(\gamma_{1}-\gamma_{0}\right)^{2}$ 


$$
\begin{aligned}
& 2 \Pi_{2}=k_{4}\left(x_{2}-x_{0}\right)^{2}+k_{5}\left(z_{2}-z_{0}\right)^{2}+k_{6}\left(y_{2}-y_{3}-y_{0}\right)^{2} \\
& +c_{5}\left(\alpha_{2}-\alpha_{0}\right)^{2}+c_{4}\left(\beta_{2}-\beta_{0}\right)^{2}+c_{6}\left(\gamma_{2}-\gamma_{0}\right)^{2}
\end{aligned}
$$

where, $x_{0}, y_{0}, z_{0}, \alpha_{0}, \beta_{0}, \gamma_{0}$ are the amplitudes of kinematical excitations acting from the foundation in the direction of the corresponding coordinate axis. follows:

Dissipative functions can be expressed as

$$
\begin{aligned}
& 2 \Phi_{1}=h_{1}\left(\dot{x}_{1}-\dot{x}_{0}\right)^{2}+h_{2}\left(\dot{z}_{1}-\dot{z}_{0}\right)^{2}+h_{3}\left(\dot{y}_{1}-\dot{y}_{0}\right)^{2} \\
& +r_{2}\left(\dot{\alpha}_{1}-\dot{\alpha}_{0}\right)^{2}+r_{1}\left(\dot{\beta}_{1}-\dot{\beta}_{0}\right)^{2}+r_{3}\left(\dot{\gamma}_{1}-\dot{\gamma}_{0}\right)^{2} \\
& 2 \Phi_{2}=h_{4}\left(\dot{x}_{2}-\dot{x}_{0}\right)^{2}+h_{5}\left(\dot{z}_{2}-\dot{z}_{0}\right)^{2}+h_{6}\left(\dot{y}_{2}-\dot{y}_{3}-\dot{y}_{0}\right)^{2} \\
& +r_{5}\left(\dot{\alpha}_{2}-\dot{\alpha}_{0}\right)^{2}+r_{4}\left(\dot{\beta}_{2}-\dot{\beta}_{0}\right)^{2}+r_{6}\left(\dot{\gamma}_{2}-\dot{\gamma}_{0}\right)^{2}
\end{aligned}
$$

The mathematical model of the whole carriage system is composed of the system of second order differential equations (Augustaitis 2000),

$$
[A]\{\ddot{q}\}+[B]\{\dot{q}\}+[C]\{q\}=\{Q(t)\}
$$

where, $[A],[B],[C]$ are the matrices of inertia, damping and rigidity, respectively; $\{q\},\{\dot{q}\},\{\ddot{q}\}$ are the vectors of displacements, velocities and accelerations, and $\{Q(t)\}$ is the vector of external forces, including forces due to kinematical excitation $\{Q(t)\}=\left\{Q_{\text {extern. }}(t)\right\}+\left\{Q_{q 0}(t)\right\}$.

Matrices $[A],[B]$ and $[C]$, vectors $\{q\},\{\dot{q}\},\{\ddot{q}\}$ and $\{Q(t)\}$ can be written as follows:

$$
\begin{gathered}
{[A]=\left[\begin{array}{cccc}
a_{11} & a_{12} & \ldots & a_{13} \\
a_{21} & a_{22} & \ldots & a_{213} \\
\vdots & \vdots & \vdots & \vdots \\
a_{131} & a_{132} & \ldots & a_{1313}
\end{array}\right] \quad[B]=\left[\begin{array}{llll}
b_{11} & b_{12} & \ldots & b_{113} \\
b_{21} & b_{22} & \ldots & b_{213} \\
\vdots & \vdots & \vdots & \vdots \\
b_{131} & b_{132} & \ldots & b_{1313}
\end{array}\right]} \\
{[C]=\left[\begin{array}{cccc}
d_{11} & d_{12} & \ldots & d_{113} \\
d_{21} & d_{22} & \ldots & d_{213} \\
\vdots & \vdots & \vdots & \vdots \\
d_{131} & d_{132} & \ldots & d_{1313}
\end{array}\right]}
\end{gathered}
$$

$$
\{q\}=\left\{\begin{array}{c}
q_{1} \\
q_{2} \\
\vdots \\
q_{13}
\end{array}\right\}\{\dot{q}\}=\left\{\begin{array}{c}
\dot{q}_{1} \\
\dot{q}_{2} \\
\vdots \\
\dot{q}_{13}
\end{array}\right\}\{\ddot{q}\}=\left\{\begin{array}{c}
\ddot{q}_{1} \\
\ddot{q}_{2} \\
\vdots \\
\ddot{q}_{13}
\end{array}\right\}
$$

$y_{3}-y_{1}-l_{2} \gamma_{1}-l_{2} \alpha_{1}=0$

The data required for modeling the rigidity of the aerostatic bearings of the carriages, the driver system connecting the force carriage with the frame, and the elements connecting the force carriage with the precision carriage, as well as amplitudes of excitation of the frame rising due to vibrations of the foundation was determined experimentally. For this purpose, vibrations excited by the impact hammer at the characteristic points of both carriages were analyzed, and vibrations of the frame supports were measured and analyzed.

The damping coefficients were evaluated as a result of the viscous friction for the entire model. Additionally, for the connection of the force and precision carriages, it was assumed that the friction force is proportional to the displacement.

Simulation and analysis of the model were carried out using Simulink and MATLAB software (The MathWorks, Inc., Natick, Massachusetts, USA). The $y$ axis is extremely important for the accuracy of the comparator because this axis coincides with the measuring direction during the scale calibration process.

Figure 4 illustrates a system response to the most important excitation-external force applied to the force carriage. Two resonance zones can be distinguished in Fig. 4: a $20-25$ and $280-310 \mathrm{~Hz}$ frequency interval.

Modes of vibrations at resonant frequencies are presented in Fig. 5. It can be seen that oscillations along coordinates $y_{1}$ and $y_{2}$ are counteracted at these frequencies.

\section{Conclusions}

1. A created dynamic model of the carriage system of the comparator allowed an analysis of its dynamic characteristics as well as their influence on calibration accuracy.

2. Two harmful resonant frequencies of the system-24 Hz and $296 \mathrm{~Hz}$-were determined.

3. Vibrations of the precision and force carriages at resonant frequencies act in opposite directions. 


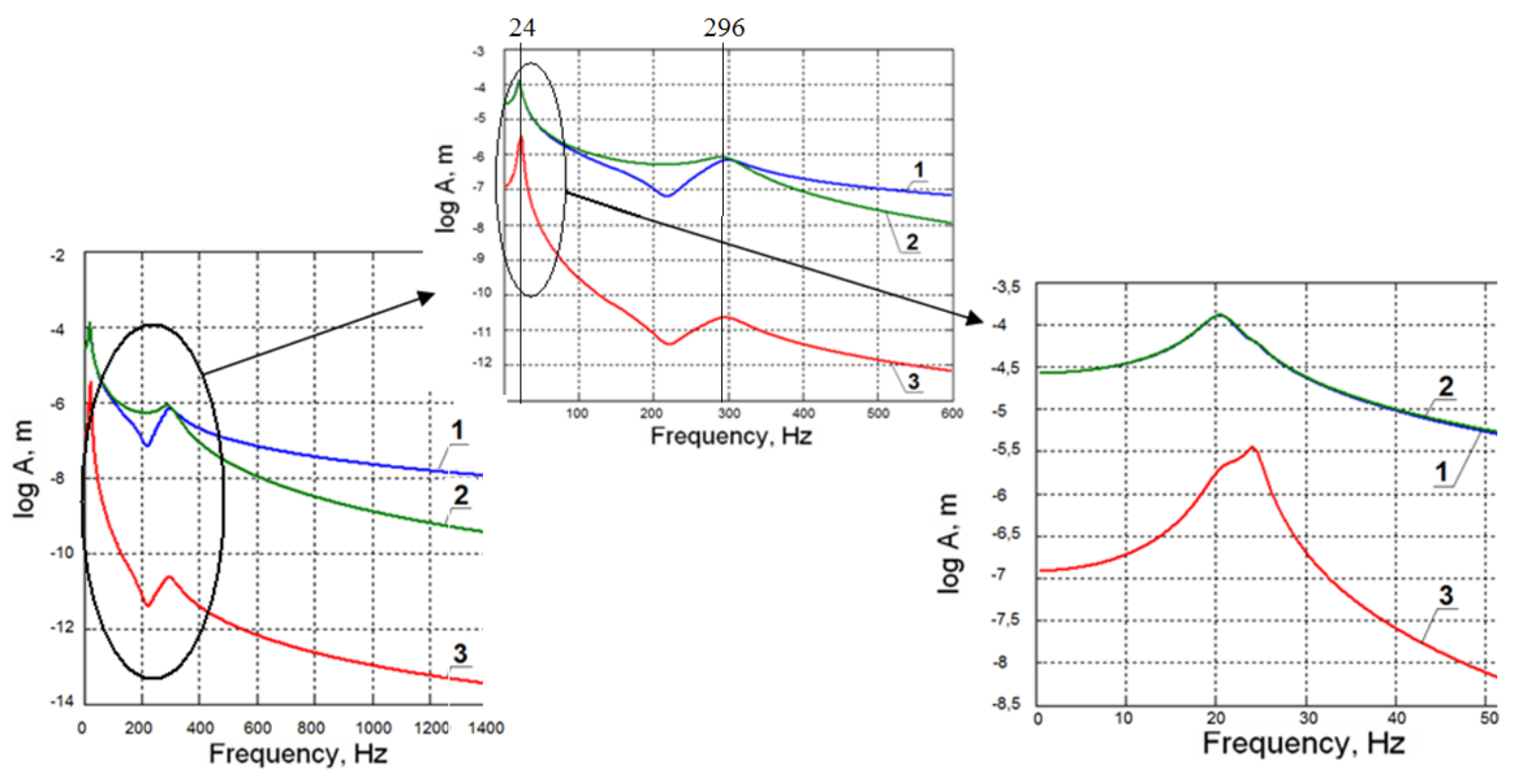

Figure 4. Amplitude-frequency response to the excitation applied to the force carriage in the $y_{2}$ direction. Line 1 indicates a precision carriage along the coordinate $y_{1}$, while line 2 indicates a force carriage along the coordinate $y_{2}$, and line 3 shows the granite frame along the coordinate $y_{0}$.
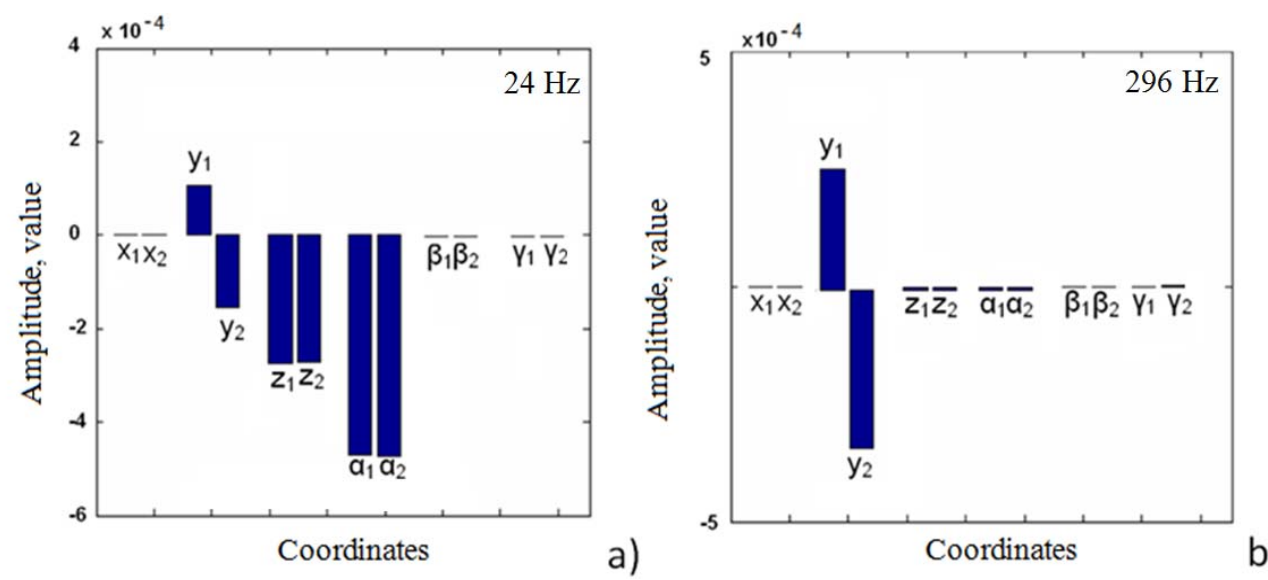

Figure 5. Modes of vibrations of the carriage system at resonant frequencies of (a) $24 \mathrm{~Hz}$ and (b) $296 \mathrm{~Hz}$.

\section{References}

Augustaitis VK (2000), Fundamentals of mechanical vibrations. Vilnius, Žiburio leidykla.

Beers JS, Penzes WB (1999), The NIST length scale interferometer. Journal of Research of the National Institute of Standards and Technology 104(3): 225252.

Bosse H, Flugge J (2002), Requirements and recent developments in high precision length metrology. NW-Verlag.
Demarest F (1998), High-resolution, high-speed, low data age uncertainty, heterodyne displacement measuring interferometer electronics. Measurement Science and Technology 9(7): 1024-1030.

Evolving needs for metrology in trade, industry and society and the role of the BIPM (2003), Bureau International des Poids et Measures (BIPM). 
Jakstas A, Kausinis S, Barauskas R, Kasparaitis A, Barakauskas A (2011), Investigation of dynamicsinduced errors of long line scale calibration systems. Measurement 44(5): 976-987.

Jakstas A, Kausinis S, Barauskas R, Kasparaitis A, Barakauskas A (2014), Thermal error analysis in precision length measurements. Measurement 51: 133-146.

Kasparaitis A, Kilikevičius A, Barakauskas A, Mokšin V (2012), Experimental research of vibrations of angle measurement comparator. Journal of Vibro Engineering 14(4): 1760-65.

Kasparaitis A, Kilikevičius A, Véžys J, Prokopovič V, Makarskas V (2015), Dynamic research on precision angle measurement comparator. Solid State Phenomena 220-221: 554-557.

Kasparaitis A, Vekteris V, Kilikevičius A (2008), Line scale comparator carriage vibrations during dynamic calibration. Journal of Vibro Engineering 10(3): 347-354.

Kaušinis S, Kasparaitis A, Barakauskas A, Barauskas R, Jakštas A, Kilikevičius A (2009), Line scale calibration in non-ideal measurement situation. Solid State Phenomena 147-149: 682685.

Kilikevičius A, Sabaitis D (2009), Stability of length measurement comparator's carriage under artificial excitation. Ultragarsas 63(1): 33-37.
Kilikevičius A, Vekteris V, Kasparaitis A (2009), Precision measurement of the carriage vibrations at the different speeds. Solid State Phenomena 147-149: 690-695.

Nakazava H (1994), Principles of precision engineering. Oxford, Oxford University Press.

Nano4: 1D gratings. (2000), Final report, Swiss Federal Office of Metrology (METAS).

Nanoscience and nanotechnologies: opportunities and uncertainties (2004), The Royal Society and The Royal Academy of Engineering (RAENG).

Sawabe M, Maeda F, Yamaryo Y, Simomura T, Saruki Y, Kubo T, Sakai H, Aoyagi S (2004), A new vacuum interferometric comparator for calibrating the fine linear encoders and scales. Precision Engineering 28(3): 320-328.

Swyt DA (2001), Length and dimensional measurements at NIST. Journal of Research of the National Institute of Standards and Technology 106(1): 1-23.

Thalmann R (1997), A new high precision length measuring machine, progress in precision engineering and nanotechnology. Proceedings of the 9-IPES/UME4, Braunschweig, Germany 1: 112-115.

Webb CE, Jones, JDC (2004), Handbook of laser technology and applications. New York, Taylor and Francis. 